\title{
Resumptive elements aid comprehension of object relative clauses: evidence from Persian*
}

\author{
RAMIN RAHMANY AND HAMIDEH MAREFAT \\ University of Tehran
}

AND

\author{
EVAN KIDD \\ Australian National University
}

(Received 23 August 2012 -Revised I 8 November 2012-Accepted 9 February 2013First published online 7 August 2013)

\begin{abstract}
The current study investigated the role of resumption in the interpretation of object relative clauses (RCs) in Persian-speaking children. Sixty-four $(N=64)$ children aged $3 ; 2-6 ; 0(M=4 ; 8)$ completed a referent selection task that tested their comprehension of subject $\mathrm{RCs}$, gapped object RCs, and object RCs containing either a resumptive pronoun or an object clitic. The results showed that the presence of a resumptive element (pronoun or clitic) had a facilitative effect on children's processing of object RCs. In both cases object RCs with resumptive elements were interpreted more accurately than gapped subject and object RCs, suggesting that resumptive elements ease processing burden in syntactically complex contexts because they provide local cues to thematic role assignment.
\end{abstract}

\section{INTRODUCTION}

Relative clauses (RCs) have been studied extensively in developmental and adult psycholinguistics (Gibson, I998; Kidd, 20I I). A common theme in

[*] This research was generously supported by a grant from the University of Tehran (grant \# 460 Iо I I/I/5). We thank Inbal Arnon, Ludovica Serratrice, and two anonymous reviewers for helpful comments on a previous version of this paper. Address for correspondence: Dr Ramin Rahmany, Faculty of Foreign Languages and Literatures, University of Tehran, Kargar Shomali Street, Tehran, IRAN. e-mail: ramin_rahmany@yahoo.com; Dr Evan Kidd, Research School of Psychology (Building 39), The Australian National University, Canberra o200, AUSTRALIA. tel: +61 26 I25 2147; fax: +61 26 I 250499 ; e-mail: evan.kidd@anu.edu.au 
experimental studies has been comparisons of performance on subject and object relative clauses. A well-established finding in the literature is that, with some qualification, subject RCs such as (I) are acquired earlier and are easier to process than object RCs, as in (2) (e.g., Diessel \& Tomasello, 2000, 2005; Gibson, I998).

(I) The horse that _ kissed the cow.

(2) The horse that the cow kissed _.

Numerous formal and functional explanations have been put forward to explain this result. Formal syntactic approaches predict a universal subject-over-object preference in RC acquisition (e.g., Friedmann, Belletti \& Rizzi, 2009). Following Chomskyan theory (Chomsky, I 995), the approach assumes that syntactic derivation does not differ qualitatively across languages; therefore the subject-over-object advantage is predicted to hold cross-linguistically.

Alternatively, processing accounts predict that comprehension is the outcome of the integration of multiple constraints on interpretation, both syntactic and non-syntactic (Bates \& MacWhinney, I989; Gennari \& MacDonald, 2008; O'Grady, 20II). These accounts argue that object RCs like (2) are difficult in languages such as English because they are distributionally infrequent and as such incur a large processing cost. Consistent with this argument, studies that have tested children on distributionally frequent object RCs report no or an attenuated subject-object asymmetry (e.g., Arnon, 2010; Brandt, Kidd, Lieven \& Tomasello, 2009; Kidd, Brandt, Lieven \& Tomasello, 2007). Operationalizing complexity with respect to distribution predicts cross-linguistic differences in acquisition. Data from Basque (Gutierrez-Mangado, 20 I I), Cantonese (Chan, Matthews \& Yip, 20 I I; Yip \& Matthews, 2007), Japanese (Ozeki, 20 I I ; Suzuki, 20 I I), and Quechua (Courtney, 2006) show that children acquiring these languages either exhibit a clear preference for object RCs or do not experience any difference in difficulty between subject and object RCs, suggesting that the subject-object asymmetry is not universal.

In the current study we investigated Persian-speaking children's comprehension of subject and object RCs. Persian is a null-subject head-final language with Subject-Object-Verb (SOV) word order (Karimi, 2005). Persian RCs are post-nominal, only allow gaps in subject RCs (3), but allow either a gap, resumptive pronoun, or object clitic in object RCs, as shown in (4)-(6) (for more general information about Persian RCs see Rahmany, Marefat \& Kidd, 20 I I).

$\begin{array}{llll}s a g-i & k e & e s b \quad r a & \text { gereft } \\ \text { dog-RM that } & \text { horse OM } & \text { grab PAST } 3 S G ~ 3 \\ \text { 'The dog that grabbed the horse' }\end{array}$




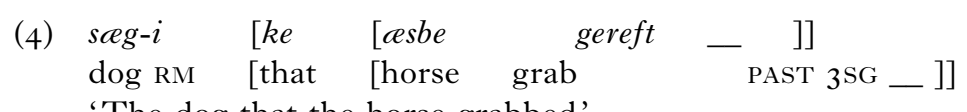

'The dog that the horse grabbed'

(5) $s a g-i \quad[k e \quad[$ esbe $u \quad$ ra gereft $]]$ dog-RM [that [horse it-RESUMPTIVE PRONOUN OM grab PAST 3SG]] 'The dog that the horse grabbed'

(6) $s a g-i \quad[k e \quad[$ aesbe gereft-eš $]]$

dog-RM [that [horse grab PAST $3 \mathrm{SG}-$ it RESUMPTIVE CLITIC ] 'The dog that the horse grabbed'

Persian RCs are introduced by a relative marker (RM) $-i$ attached to the head noun, as in (3). Persian does not have relative pronouns: the RC is always introduced by the relative complementizer ke. Thus the complementizer is invariant; it takes the same form regardless of the animacy, gender, grammatical function, or number of the head. Resumptive pronouns and clitics are marked for number, and in ${ }_{3} \mathrm{PS}$ contexts resumptive pronouns make an animate/inanimate distinction (i.e., s/he vs. it). Finally, in Persian verbs are inflected for number and person, and specific objects are marked by $r \hat{a}, r o$, or $o(\mathrm{OM}=$ object marker $)$. The $\mathrm{OMs}$ are restricted in distribution: they are obligatory in gapped subject RCs and in object RCs containing resumptive pronouns, but are ungrammatical in gapped object RCs and object RCs containing a resumptive clitic. The three forms are allomorphs: $r \hat{a}$ is used in the formal register; ro and $o$ are used in colloquial speech.

Persian RCs are interesting for two reasons. First, gapped subject and object RCs constitute a minimal pair distinguished only by the overt OM in subject RCs. This means that children must delay thematic role assignment until they identify the presence/absence of the OM. Since both arguments come before the verb, several processing theories predict no subject-object asymmetry (Gibson, I 998; O’Grady, 20 I I). Second, Persian allows optional resumptive pronouns and clitics in object $\mathrm{RCs}$, which processing approaches predict facilitate comprehension (Arnon, 2005). We next consider this latter prediction in more detail.

\section{Resumptives in the acquisition of RCs}

Early discussions on the role of resumption in acquisition focused on whether or not their presence in children's productions of non-subject RCs were indicative of a problem with movement in child grammar (Guasti \& Shlonsky, I995; Labelle, I990; Pérez-Leroux, I995; see also Friedmann et al., 2009). The results of other studies suggest that resumptives might serve a functional purpose; specifically, they might allow children to track 
and assign thematic roles in syntactically complex contexts, an effect which may have motivated their use even in languages where they are considered to be ungrammatical. We review this evidence below.

McKee and McDaniel (200I) reported on three studies that investigated English-speaking children's and adults' production and comprehension of a range of sentences containing resumptive pronouns. A detailed description of the study is beyond the scope of this paper; however, two results are relevant. First, children aged 3;5-8; I I PRODUCED resumptive pronouns in object RCs (and other complex syntactic environments) at significantly higher rates than adults. Similar effects were observed in a grammaticality judgement task. McKee and McDaniel argued that the effects were due to developmental differences in parsing, suggesting that children 'shunt' clauses out of active memory earlier than do adults, and therefore produce and accept resumptives at a greater rate in order to reactivate a head. Recent on-line processing research has reported similar effects for English-speaking adults, suggesting that resumptives continue to alleviate complexity in the adult system (Hofmeister \& Norcliffe, 2013).

Arnon (2005) investigated four- to five-year-old Hebrew-speaking children's comprehension and production of subject and object RCs using both comprehension (picture-pointing) and elicited production. Unlike in English, the use of resumptive pronouns in object RCs is a permissible (although infrequent) grammatical option in Hebrew. Arnon observed an intriguing pattern of results across the two tasks: those children who produced the most resumptive pronouns in the elicitation task (in $80 \%$ of all object RC contexts) performed almost at floor on gapped object RCs in the comprehension task ( $5 \%$ correct). Across the entire sample, the tendency to use resumptives strongly correlated with errors in comprehension $(r=0.57)$. A small follow-up study tested children $(N=7)$ who performed poorly on the comprehension component of the main study by directly comparing the comprehension of gapped object RCs and object RCs containing resumptive pronouns using picture-pointing. The presence of a resumptive pronoun doubled the children's correct responses and significantly reduced their tendency to make errors of thematic role assignment. For instance, the presence of a resumptive pronoun significantly reduced the tendency for children to interpret the granny in the granny that the girl kisses as the agent rather than the patient. Overall, these data suggest that the use of resumptive pronouns in production is associated with difficulty comprehending gapped object RCs, whereas their presence in the input facilitates comprehension by aiding thematic role assignment.

Overall, past results suggest that resumptives might serve a useful function, helping children to reliably monitor participant roles in production, as well as alleviating the traditional difficulty associated with long-distance dependencies in comprehension. The current study investigated three- to 
six-year-old Persian-speaking children's comprehension of (i) gapped subject and object RCs, as well as object RCs that contained either (ii) a resumptive pronoun or (iii) an object clitic. Two comparisons were of interest. First, our primary aim was to determine whether resumption facilitates comprehension of object RCs. Following Arnon (2005), we predicted that object RCs with resumptive pronouns and clitics would be comprehended significantly better than gapped object RCs. A secondary aim was to compare children's comprehension of gapped subject and object RCs. Processing theories that define complexity as the linear distance between verbs and their arguments predict no asymmetry in Persian, since gapped subject and object RCs have the same NNV word order (Gibson, I998; O’Grady, 20 I I). Previous work on Persian reported a subject advantage in acquisition (Rahmany et al., 20II); however, this previous study did not present the test sentences with a supportive discourse context, which significantly affects children's performance (see Corrêa, I 995). In the current study we presented sentences to children in a felicitous discourse context; we therefore predicted no subject advantage.

\section{METHOD}

\section{Participants}

Sixty-four $(N=64,39$ female) monolingual Persian-speaking children aged $3 ; 2-6 ; \circ(M=4 ; 8, S D=0 ; 8)$ were recruited from a nursery school in Tehran. This age range was chosen because past studies have shown that children of this age are likely to both frequently use resumptives and benefit from their presence in comprehension (Arnon, 2005; McKee \& McDaniel, 200I). All participants were typically developing with no noted language impairments, hearing deficits, neurological difficulties, or social, emotional, or behavioral problems.

\section{Materials and procedure}

The children were tested in a quiet room of their nursery using the referent selection task developed by Brandt et al. (2009). They were introduced to pairs of toy animals (bear, horse, elephant, cow, dog), which were distinguishable by color (e.g., a brown dog and a white dog). During a warm-up session the children were asked to name each animal type; all children knew every animal.

The referent selection task presents two narrated background scenes to children that provide a felicitous discourse context for use of a restrictive relative clause (for a discussion of the importance of context, see Corrêa, I 995 ; Kidd, 2003). In the current study the background scenes also provided a felicitous context for the use of resumptives, since their status as 
pronominal elements requires that they have antecedents in discourse. For example, in one item there was a dog, a cat, and two horses. The background scenes proceeded as in (7).

(7) 'The dog kisses this horse. The dog pushes the other horse.'

The experimenter simultaneously narrated and acted out each background scene. They were immediately followed by a distractor scene; for example, 'Now the dog goes swimming'. The function of the distractor scene was to provide a buffer between the background scenes and tests scenes, which served to minimize recency effects when children were required to choose between the two tokens of the head upon hearing the test sentence. The test sentence was then presented, as in (8).

(8) 'Can you give me the horse that the dog kissed?'

The child's task was simply to choose the appropriate token of the head referent, thereby demonstrating that they had processed the $\mathrm{RC}$ as a noun modifier. The experimenter repeated the test sentence once if the child did not pick up or unambiguously point to a referent. After one repetition the experimenter continued with the next item. The referents and their positions were counterbalanced so that half of the time the target referent was introduced in the first background scene and the other half of the time it was introduced in the second background scene. Similarly, half of the time the target appeared on the left side of the table and the other half of the time it appeared on the right side. In addition, the order of presentation was pseudo-randomized such that test sentences from the same condition did not appear twice in a row. The verbs used were 'wash', 'pull', 'grab', 'hit', and 'kiss', all of which are one-part Persian verbs (i.e., no compound verbs were used). The task consisted of twenty-one items (4 subject RCs, 4 gapped object RCs, 4 object RCs containing a resumptive pronoun, 4 object RCs containing a resumptive clitic, and 5 fillers).

\section{RESULTS}

Each response was coded as correct or incorrect. Table I shows the children's average performance on each structural type. Overall, the children performed similarly on subject RCs and gapped object RCs, but their performance on both object RCs with a resumptive pronoun and an object clitic was approximately 10\% higher than both gapped structures.

The data were analyzed using Generalized Linear Mixed Models (GLMM) (Jaeger, 2008), which were calculated using the lme 4 package for Linear Mixed Effects (Bates \& Maechler, 2010) in $R$ (version 2.I4.2; R Core Development Team, 2008). Structural type (4 levels : subject, object, object-resumptive pronoun, object-object clitic) and age (in months) were 
RESUMPTION IN PERSIAN RELATIVE CLAUSES

TABLE I. Mean proportion correct and standard deviation for each structural type

\begin{tabular}{lcccc}
\hline & \multicolumn{4}{c}{ Structure } \\
\cline { 2 - 5 } & Subject RC & Object RC & Object-Res. & Object-Clitic \\
\hline Mean & $\cdot 496$ & $\cdot 508$ & $\cdot 62$ & $\cdot 59$ \\
SD & 5 I & $\cdot 5$ & $\cdot 49$ & $\cdot 49$ \\
\hline
\end{tabular}

NOTE: $N=64$.

included as fixed effects. Structural type was centered with a mean of $\circ$ and range of $\mathrm{I}$; age was mean centered. Participants and items were included as random effects in order to account for by-subject and by-item variation. Additionally, by-subject random slopes were included for structure type to control for individual variability in performance across conditions. Both random effects and the by-subject random slopes significantly contributed to model fit. However, the structure by age interaction term did not and was therefore removed $\left(\chi^{2}(3)=75, p=\cdot 86\right)$. The final model is shown in Table 2 .

Table 2 shows a positive main effect of age, reflecting the fact that accuracy increased with age. The children did not differ in their performance on gapped subject and object RCs; however, they were significantly more accurate on object RCs with either a resumptive pronoun or an object clitic than on subject RCs. Subsequent analyses showed that the children's performance on object $\mathrm{RCs}$ with resumptives was significantly better than their performance on gapped object $\operatorname{RCs}\left(\beta=0 \cdot 5 \mathrm{I}, z=2 \cdot 66, p=\cdot{ }^{\circ}, 8\right)$. The difference between object RCs containing a resumptive clitic and gapped object RCs was marginal in the same direction $(\beta=0 \cdot 35, z=\mathrm{I} \cdot 92$, $p=\cdot 055)$. Performance on object $\mathrm{RCs}$ with resumptive pronouns did not differ from performance on object RCs with resumptive clitics $(\beta=0 \cdot 16$, $z=84, p=\cdot 4)$.

\section{Error analysis}

Children tend to make two errors in the referent selection task: (i) 'Head Token' errors, where they choose the incorrect token of the head referent, and (ii) 'NP-other' errors, where they select the non-head NP-referent in the sentence (i.e., the sheep, in 'the horse that the sheep bumped'). The former error was most prevalent, accounting for $34.8 \%$ of responses; the latter error type occurred less often, accounting for only $5 \cdot 7 \%$ of total responses. These frequencies are similar to error rates in English- and German-speaking children (Brandt et al., 2009). Table 3 shows the mean proportion of both error types by condition. 
RAHMANY ET $A L$.

TA B LE 2. Summary of the final model (reference level for fixed effect of structure type : subject $R C$ )

\begin{tabular}{lcccc}
\hline Predictor & $\beta$ & $S E$ & $z$ & $p$ \\
\hline Intercept & -0.199 & .23 & -0.87 & .39 \\
Structure(Object) & 0.200 & 24 & $0.8 \mathrm{I}$ & .42 \\
Structure(Object Res.) & 0.964 & 28 & 3.49 & $<.00 \mathrm{I}$ I* $^{*}$ \\
Structure(Object Clitic) & 0.637 & .29 & $2.2 \mathrm{I}$ & $.027^{*}$ \\
Age (months) & 0.03 & $.0 \mathrm{I}$ & $2.9 \mathrm{I}$ & $.003^{* *}$ \\
\hline
\end{tabular}

NOTES: $* p<\cdot 05, * * p<\cdot 01, * * * *<\cdot 00 \mathrm{I} ; \log$ likelihood $=-666 \cdot \mathrm{I}$.

TABLE 3. Mean proportion of error types by condition (SD in brackets)

\begin{tabular}{lcccc}
\hline & \multicolumn{4}{c}{ Structure } \\
\cline { 2 - 5 } & Subject RC & Object RC & Object-Res. & Object-Clitic \\
\hline Head Token & $\cdot 4 \mathrm{I}(\cdot 49)$ & $.4 \mathrm{I}(\cdot 49)$ & $.29(\cdot 46)$ & $.28(\cdot 45)$ \\
NP Other & $\cdot 04(\cdot \mathrm{I} 9)$ & $.05(\cdot 23)$ & $.07(\cdot 25)$ & $.07(\cdot 25)$ \\
\hline
\end{tabular}

NOTE : $N=64$.

Table 3 shows that children made fewer Head Token errors on object $\mathrm{RCs}$ with resumptive elements than on gapped subject and object RCs. The distribution of NP Other errors was flatter. The data were again analyzed using GLMMs. For Head Token errors the results were almost identical to the accuracy data, except that the main effect of age was marginal and was therefore removed $(\beta=-0 \cdot 12, z=-\mathrm{I} \cdot 68, p=\cdot 09)$. The difference between gapped subject and object RCs was not significant $(\beta=-0.02, z=-0.09$, $p=\cdot 93$ ), but children made fewer Head Token errors on object RCs containing either a resumptive pronoun or a resumptive clitic than on both subject RCs (Obj. Res. vs. Subject RC: $\beta=-0 \cdot 58, z=-3 \cdot 09, p=\cdot 002$; Obj. Clitic vs. Subject RC: $\beta=-0.52, z=-2 \cdot 8, p=\cdot 005)$ and gapped object RCs (Obj. Res. vs. Object RC: $\beta=-0 \cdot 57, z=-3, p=\cdot 003$; Obj. Clitic vs. Object RC: $\beta=-0.5 \mathrm{I}, z=-2.7 \mathrm{I}, p=.007)$. The difference between the two types of object RCs with resumption was not significant $(\beta=-0.06$, $z=-0.30, p=\cdot 77)$. The analysis of the NP Other errors yielded no significant effects.

\section{DISCUSSION}

Both hypotheses were supported: resumption significantly facilitated comprehension of object RCs, and we observed no subject-object asymmetry in gapped subject and object RCs. Each result is discussed in turn. 
First, the pattern of results suggests that resumption reduces the ambiguity associated with thematic role assignment, thus improving children's performance. This interpretation is supported by both the accuracy and error data. Specifically, resumption significantly reduced the tendency to make head token errors, where children selected the incorrect token of the head referent. This error type is not strictly an error of thematic role assignment; however, its reduction in resumptive contexts provides an indication of how resumption might be important for this process. Recall that gapped Persian RCs require thematic role assignment to be delayed until the children can identify the presence or absence of the OM before the verb. Object RCs with resumptive pronouns circumvent this problem by placing the resumptive pronoun before the $\mathrm{OM}$; object $\mathrm{RCs}$ with resumptive clitics reduce any residual ambiguity at the verb by providing an additional overt cue. Both elements provide LOCAL cues to grammatical role assignment. This interpretation is consistent with Arnon (2005), who reported that resumptive pronouns in Hebrew object RCs both increased accuracy and reduced errors involving thematic role assignment. The results support processing-based interpretations of sentence difficulty in acquisition (Arnon, 20 ro; Kidd et al., 2007; O’Grady, 20I I ).

It appears that resumptive elements facilitate comprehension despite the fact that resumption is not a frequently used strategy in naturalistic speech. We coded roo RCs extracted from the speech of Minu (aged 4;9-5;2) from the Family Farsi corpus (Family, 2009), as well as roo RCs identified in his caregiver's speech from the same period (available on CHILDES; MacWhinney, 2000). This period was chosen because it approximately maps onto the mean age of our sample. Gapped RCs were frequent: Minu produced more subject than object gapped RCs $(47 \%$ versus $33 \%$ ); the distribution of gapped $\mathrm{RCs}$ in the input was more even ( $38 \%$ subject, $42 \%$ object). Strikingly, no object RCs contained resumptive pronouns, and very few contained object clitics (Minu $=4 \%$; Input $=3 \%$ ). The four instances of resumptive clitics in Minu's speech all occurred in instances where there was a long distance between the head noun and the gap, supporting our contention that resumption helps speakers track participant roles in syntactically complex contexts. It is notable that there were few resumptive elements in CDS. Given that resumption aids children's comprehension, their infrequent use by caregivers suggests that they are not used for the benefit of the language learner. Instead, they seem to be used entirely for the benefit of the speaker; their function as a comprehension aid might be an unintended by-product of this production strategy.

Consistent with our second hypothesis, we did not observe a difference between subject and gapped object RCs. This result is predicted for Persian by processing theories that compute complexity on the basis of the linear 
order of arguments and their distance from verbs (e.g., Gibson, I998; O'Grady, 20II). However, it is unclear from our data whether this result is due to the unique structural properties of Persian, or because our method presented the test sentences within a supportive discourse context. A previous acquisition study did find a subject advantage for Persian (Rahmany et al., 20I I), but this study used the arguably more difficult picture-pointing technique, and did not present the test sentences in a supportive discourse context. Thus, it is possible that the use of a supportive discourse context in the current study aided the children's processing of gapped object RCs. Although the subject-object asymmetry is consistently observed when children are not tested on sentences presented in a felicitous discourse context, the presence of a supporting context has been shown to eliminate the subject-object asymmetry in past studies. For instance, Kidd and Bavin (2002) did not observe a significant effect of extraction site in right-branching RCs in English-speaking children aged three to five years. Similarly, Brandt et al. (2009), who used the same referent selection task used in the current study, observed no difference between subject and object RCs that contained two animate NPs in young three-year-old English-speaking children, but did in German-speaking children of the same age. Furthermore, Roland, Mauner, O'Meara, and Yun (2012) recently reported that a supporting discourse context neutralized the subject-object asymmetry in adults. Therefore, the result is not without precedent, but has not received as careful empirical scrutiny in the literature as perhaps it should (see Weighall, 2008). Since some accounts of the acquisition of RCs are crucially hinged upon the expectation that certain types of object RCs will ALWAYs be more difficult than subject RCs (e.g., Friedmann et al., 2009; cf. Goodluck, 20 Io), determining the exact influence of discourse context is a priority for future research.

\section{CONCLUSION}

Consistent with past acquisition research on Hebrew (e.g., Arnon, 2005), we have shown that resumptive elements significantly improve Persian-speaking children's comprehension of object RCs. Additionally, we did not observe a subject-object asymmetry in gapped subject and object RCs, a result that could be due to unique structural properties of Persian as well as the method we employed. We argued that resumptives serve a disambiguating role in comprehension, acting as a local cue to thematic role assignment. Since resumptives also facilitate on-line processing of complex structures in adults, we suggest that the result is best explained as a parsing effect, supported processing based accounts of syntactic acquisition (e.g., Bates \& MacWhinney, I989; O’Grady, 20 I I).

\section{6}




\section{REFERENCES}

Arnon, I. (2005). Relative clause acquisition in Hebrew: towards a processing-oriented account. In A. Brugos, M. Clark-Cotton \& S Ha (eds.), Proceedings of the 29th Annual Boston Conference on Language Development, 37-48. Somerville, MA: Cascadilla Press.

Arnon, I. (20I0). Re-thinking child difficulty: the effect of NP-type on children's processing of relative clauses in Hebrew. Fournal of Child Language 37, $27-57$.

Bates, D. \& Maechler, M. (2010). lme4: Linear mixed effects models using $\mathrm{S}_{4}$ classes. R package. 999375-33.

Bates, E. \& MacWhinney B. (I989). Functionalism and the Competition Model. In B. MacWhinney \& E. Bates (eds.), The crosslinguistic study of sentence processing, 3-76. New York: Cambridge University Press.

Brandt, S., Kidd, E., Lieven, E. \& Tomasello, M. (2009). The discourse bases of relativization: an investigation of young German- and English-speaking children's comprehension of relative clauses. Cognitive Linguistics 20, 539-70.

Chan, A., Matthews, S. \& Yip, V. (20II). The acquisition of relative clauses in Cantonese and Mandarin. In E. Kidd (ed.), The acquisition of relative clauses: processing, typology, and function, 197-226). Amsterdam: John Benjamins.

Chomsky, N. (1 995). The Minimalist Program. Cambridge, MA: MIT Press.

Corrêa, L. M. S. (I 995). An alternative assessment of children's comprehension of relative clauses. Fournal of Psycholinguistic Research 24, I 83-203.

Courtney, E. H. (2006). Adult and child production of Quechua relative clauses. First Language 26, 3 I 7-38.

Diessel, H. \& Tomasello, M. (2000). The development of relative clauses in spontaneous child speech. Cognitive Linguistics II, I 3 I-5 I.

Diessel, H. \& Tomasello, M. (2005). A new look at the acquisition of relative clauses. Language 81, 882-906.

Family, N. (2009). Lighten up: the acquisition of light verb constructions in Persian. In J. Chandlee, M. Franchini, S. Lord \& G. Rheiner (eds.), Proceedings of the 33rd Annual Boston University Conference on Language Development, 139-50. Somerville, MA: Cascadilla Press.

Friedmann, N., Belletti, A. \& Rizzi, L. (2009). Relativized relatives: types of intervention in the acquisition of A-bar dependencies. Lingua 119, 67-88.

Gennari, S. P. \& MacDonald, M. C. (2008). Semantic indeterminacy in object relative clauses. Fournal of Memory and Language 58, i6 6 - -87 .

Gibson, E. (1998). Linguistic complexity: locality of syntactic dependencies. Cognition 68, I-76.

Goodluck, H. (20 Io). Object extraction is not subject to Child Relativized Minimality. Lingua I20, I 5 I6-2I.

Guasti, M. T. \& Shlonsky, U. (1995). The acquisition of French relative clauses. Language Acquisition 4, 257-76.

Gutierrez-Mangado, M. J. (20 I I). Children's comprehension of relative clauses in an ergative language: the case of Basque. Language Acquisition 18, I76-201.

Hofmeister, P. \& Norcliffe, E. (2013). Does resumption facilitate sentence comprehension? In P. Hofmeister \& E. Norcliffe (eds.), The core and the periphery: data-driven perspectives on syntax inspired by Ivan A. Sag, I-25. Stanford, CA: CSLI Publications.

Jaeger, T. F. (2008). Categorical data analysis: away from ANOVAs (transformation or not) and towards logit mixed models. Fournal of Memory and Language 59, 434-46.

Karimi, S. (2005). A Minimalist approach to scrambling : evidence from Persian. Berlin: Mouton de Gruyter.

Kidd, E. (2003). Relative clause comprehension revisited: commentary on Eisenberg (2002). Fournal of Child Language 3o, $67 \mathrm{I}-79$.

Kidd, E. (ed.) (201 I). The acquisition of relative clauses: processing, typology, and function. Amsterdam: John Benjamins. 
Kidd, E. \& Bavin, E. L. (2002). English-speaking children's understanding of relative clauses: evidence for general-cognitive and language-specific constraints on development. Fournal of Psycholinguistic Research 3I, 599-6 I 7.

Kidd, E., Brandt, S., Lieven, E. \& Tomasello, M. (2007). Object relatives made easy: a crosslinguistic comparison of the constraints influencing young children's processing of relative clauses. Language and Cognitive Processes 22, 860-97.

Labelle, M. (I990). Predication, wh-movement and the development of relative clauses. Language Acquisition I, 95-I I9.

MacWhinney, B. (2000). The CHILDES Project: tools for analyzing talk, 3rd edn. Vol. $2:$ the database. Mahwah, NJ: Lawrence Erlbaum Associates.

McKee, C. \& McDaniel, D. (200I). Resumptive pronouns in English relative clauses. Language Acquisition 9, I I 3-56.

O'Grady, W. (20I I). Relative clauses: processing and acquisition. In E. Kidd (ed.), The acquisition of relative clauses: processing, typology, and function, I 3-38. Amsterdam: John Benjamins.

Ozeki, H. (201 I). The acquisition of relative clauses in Japanese. In E. Kidd (ed.), The acquisition of relative clauses: processing, typology, and function, I73-96. Amsterdam: John Benjamins.

Pérez-Leroux, A. T. (I995). Resumptives in the acquisition of relative clauses. Language Acquisition 4, $105-38$.

Rahmany, R., Marefat, H. \& Kidd, E. (20II). Persian-speaking children's acquisition of relative clauses. European Fournal of Developmental Psychology 8, 367-88.

$\mathrm{R}$ Core Development Team (2008). R: a language and environment for statistical computing. Vienna: R Foundation for Statistical Computing.

Roland, D., Mauner, C., O'Meara, C. \& Yun, H. (2012). Discourse expectations and relative clause processing. Fournal of Memory and Language 66, 479-508.

Suzuki, T. (201 I). A case-marking cue for filler-gap dependencies in children's relative clauses in Japanese. Fournal of Child Language 38, ro84-95.

Weighall, A. (2008). On still being led down the kindergarten path: children's processing of structural ambiguities. Fournal of Experimental Child Psychology 99, 75-95.

Yip, V. \& Mathews, S. (2007). The bilingual child: early development and language contact. Cambridge: Cambridge University Press. 